\title{
CINÉTICA DE ADSORÇÃO DE CORANTES ALIMENTÍCIOS EM SISTEMA BINÁRIO POR HIDROGÉL SCAFFOLD DE NANOTUBOS
}

\author{
M.M. CRISPIM ${ }^{1}$, E.C. RIOS ${ }^{1}$, K.A. SILVA ${ }^{1}$, J.O. GONÇALVES ${ }^{1}$ e L.A.A. PINTO ${ }^{1}$ \\ ${ }^{1}$ Universidade Federal do Rio Grande, Escola de Química e Alimentos \\ E-mail para contato: marsellee.martins@gmail.com
}

\begin{abstract}
RESUMO - O processo de adsorção vem ganhando destaque como alternativa na remoção dos corantes alimentícios em efluentes industriais, devido a sua simplicidade de operação e viabilidade técnica. Os hidrogéis aplicados na adsorção se tornam relevantes, devido sua estabilidade em soluções ácidas e capacidade de reuso. Por isso, o trabalho tem como objetivo desenvolver hidrogéis a base de quitosana modificados com nanotubo de carbono $(\mathrm{OH})$ e realizar o estudo cinético da adsorção dos corantes alimentícios, vermelho $\mathrm{n}^{\circ} 40 \mathrm{e}$ azul brilhante, em sistema aquoso binário. Foram realizadas análises para a caracterização do adsorvente, incluindo força do gel, grau de intumescimento e imagem de MEV. Os dados cinéticos foram ajustados aos modelos de pseudoprimeira ordem (PPO) e Avrami. Os resultaram mostraram que o hidrogel obtido apresentou alto grau de intumescimento e força de gel. As maiores capacidades de adsorção para o hidrogel foram de 239 e $214 \mathrm{mg} \mathrm{g}^{-1}$ para os corantes, azul brilhante e vermelho $n^{\circ} 40$, respectivamente. O modelo de Avrami foi o que melhor se ajustou aos dados devido aos altos valores do coeficiente de determinação $\left(R^{2}>0,95\right)$ e baixos valores do erro relativo médio $(E R M<6 \%)$.
\end{abstract}

\section{INTRODUÇÃO}

Nos dias de hoje sabe-se que o aumento do descarte de efluentes coloridos no meio ambiente provenientes das indústrias de alimentos, pode causar conflitos no ecossistema. Os corantes interferem nos processos fotossintéticos do meio aquoso, assim afetando diretamente toda a biodiversidade nela presente, além do excesso deste aditivo poder causar danos à saúde da população em geral. Por isso, se faz necessário a remoção de forma eficaz destes corantes em efluentes (Luna et al., 2018; Tan et al., 2015; Vakili et al., 2016). 


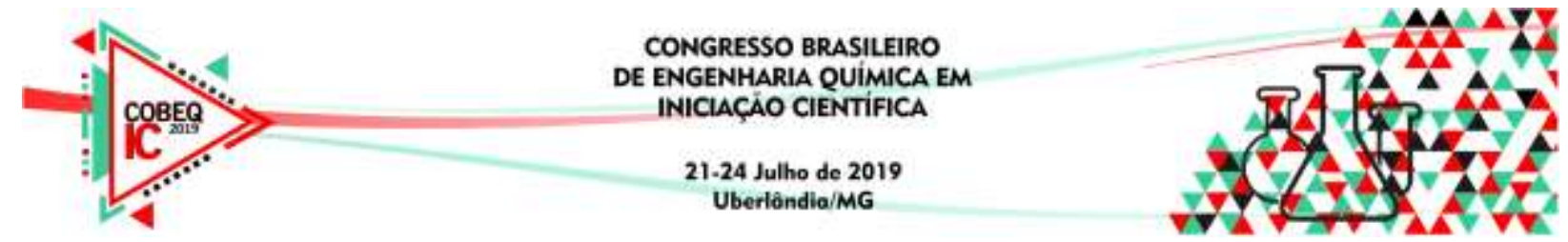

A adsorção vem ganhando notoriedade como um dos métodos mais utilizados para a remoção de corantes alimentícios, devido a seu baixo custo inicial, facilidade de operação e eficiência em relação aos métodos convencionais (Crini e Badot, 2009; Gonçalves et al., 2017). Um adsorvente promissor para a remoção de corantes em soluções aquosas é a quitosana, um polissacarídeo versátil, devido as possibilidade de modificações químicas e interações com diferentes compostos. Os hidrogéis a base de quitosana quando liofilizados tornam-se materiais secos e porosos, sendo chamados assim de scaffolds (Croisier; Jérôme, 2013; Jayakumar et al., 2011). Com o intuito de aumentar o potencial adsortivo do hidrogel e suas propriedades mecânicas, diferentes modificações podem ser feitas com relação ao adsorvente para incrementar seu desempenho.

Portanto, o objetivo deste trabalho foi obter e caracterizar hidrogel de quitosana scaffold, com a adição de nanotubo de carbono funcionalizado $(\mathrm{OH})$, e realizar o estudo cinético de adsorção dos corantes azul brilhante e vermelho $\mathrm{n}^{\circ} 40$ em sistema aquoso binário.

\section{MATERIAL E MÉTODOS}

\subsection{Obtenção do hidrogel scaffold}

A quitosana utilizada para a elaboração do hidrogel foi preparada a partir de rejeitos de camarão (Penaeus brasiliensis) segundo o método descrito por Weska et al., (2007). A quitosana obteve o grau de desacetilação de $85 \%$, foi purificada e seca em leito de jorro. $\mathrm{O}$ hidrogel foi obtido através da dissolução de quitosana $5 \%(\mathrm{~m} / \mathrm{v})$ em ácido acético $1 \%(\mathrm{v} / \mathrm{v})$. A modificação do hidrogel de quitosana foi realizada com adição $10 \%(\mathrm{~m} / \mathrm{m})$ de nanotubo de carbono $(\mathrm{OH})$. Após, foi adicionado o glutaraldeído $(1,5 \%(\mathrm{v} / \mathrm{v}))$ para formação do gel (Gonçalves et al., 2017). O hidrogel foi submetido à secagem a frio em um liofilizador (Liobras, L108, Brasil) por $48 \mathrm{~h}$, utilizando-se vácuo de $44 \mathrm{mmHg}$ e temperatura de $-54^{\circ} \mathrm{C}$.

\subsection{Caracterização do hidrogel scaffold}

Microscopia eletrônica de varredura (MEV): A imagem de alta resolução da superfície do hidrogel scaffold foi obtida a partir da análise de microscopia eletrônica de varredura (MEV), em que foi utilizado o microscópio eletrônico (Jeol, JSM6060, Japão). 


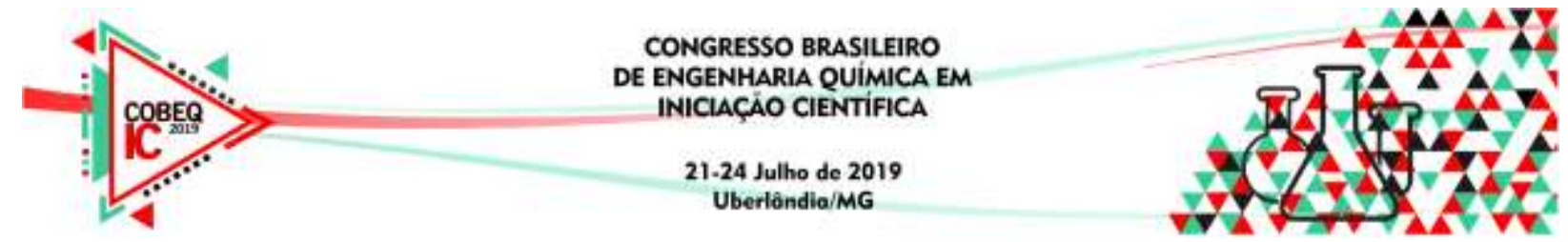

Grau de intumescimento: $250 \mathrm{mg}$ da amostra de hidrogel antes da liofilização foram colocado para inchar em água destilada onde foi agitado $(50 \mathrm{rpm})$ à $25^{\circ} \mathrm{C}$ durante 24 horas. $\mathrm{O}$ excesso de água da superfície foi removido e as amostras inchadas foram pesadas. O grau de intumescimento $(G I)$ foi determinado pela Equação 1:

$$
G I=\left(\frac{m_{i}-m_{f}}{m_{i}}\right) \times 100 \%
$$

Força do gel: A força de gel (g) do hidrogel foi medida em analisador de textura (TA.XTplus, Stable Micro Systems, Inglaterra), utilizando sonda de teflon com 12,5 mm de diâmetro pressionando $4 \mathrm{~mm}$ no hidrogel à velocidade de $1 \mathrm{~mm} \mathrm{~s}^{-1}$.

\subsection{Ensaios cinéticos}

Os experimentos cinéticos foram realizados utilizando $25 \mathrm{mg}$ do hidrogel onde foi adicionado a solução de corantes $\left(100 \mathrm{mg} \mathrm{L}^{-1}\right)$, após o pH foi ajustado em $\mathrm{pH} 3$. As amostras foram avolumadas à $1 \mathrm{~L}$ e os experimentos foram realizados em um agitador termostatizado (Jar Test, Innova 44, New BrunswickScientific, EUA). A taxa de agitação utilizada foi de 100 rpm. Alíquotas foram removidas em intervalos de tempo pré-determinados $(0,2,4,6,8,10$, $15,20,25,30,40,50,60,80,100$ e 120). A concentração dos corantes remanescente na solução foi determinada por espectrofotometria na região do visível $\left(\lambda_{\text {azul }}=610 \mathrm{~nm}\right.$; $\lambda_{\text {vermelho }}=425 \mathrm{~nm}$ ) (Quimis, Q108 DRM, Brasil). A capacidade de adsorção no tempo $t$ foi determinada através da Equação 2:

$$
\mathrm{q}_{\mathrm{t}}=\frac{\left(\mathrm{C}_{0}-\mathrm{C}_{\mathrm{t}}\right)}{\mathrm{m}} V
$$

Após, os dados cinéticos foram ajustados aos modelos de pseudo-primeira ordem e Avrami, com o intuito de melhor representar os dados.

\section{RESULTADO E DISCUSSÃO}

\subsection{Caracterização do hidrogel scaffold}

O hidrogel foi caracterizado quanto ao grau de intumescimento (GI), força de gel (FG) e imagens de MEV. Com relação à análise de $G I$, o hidrogel apresentou um valor de $91 \pm 2 \%$. Já 


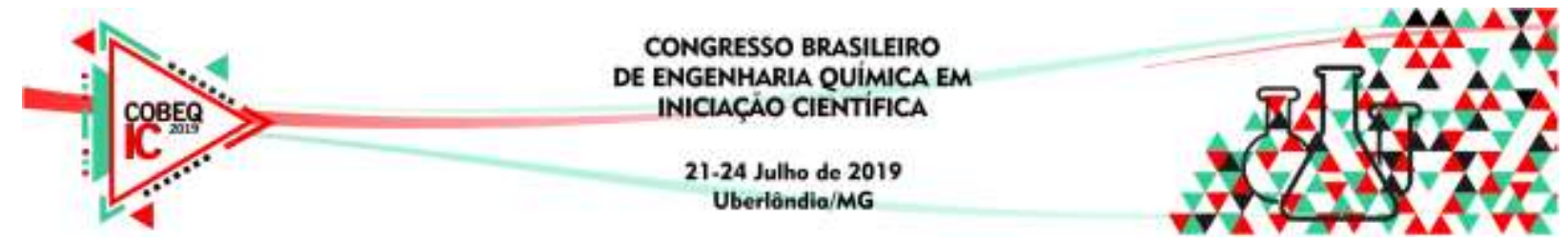

na análise de $F G$, o adsorvente apresentou um valor de $735 \pm 4 \mathrm{~g}$. Com intuito de verificar a morfologia do adsorvente após a introdução do nanotubo, foi analisada a imagem fotográfica do hidrogel scaffold, que está apresentada na Figura 1. Pode-se observar que, a superfície do hidrogel apresentou-se irregular e com características peculiares (partículas pequenas de forma cilíndrica) referentes à presença do nanotubo de carbono.

Figura 1- Imagens de MEV do hidrogel modificado com nanotubo de carbono (x5000).

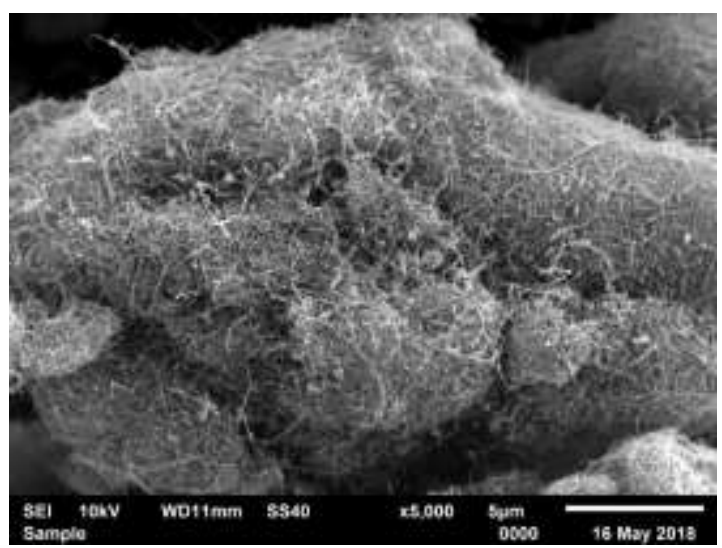

\subsection{Ensaios cinéticos}

As curvas cinéticas da adsorção dos corantes alimentícios pelo hidrogel scaffold estão apresentadas na Figura 2.

Figura 2- Curvas cinéticas de adsorção para os corantes alimentícios por hidrogel scaffold.

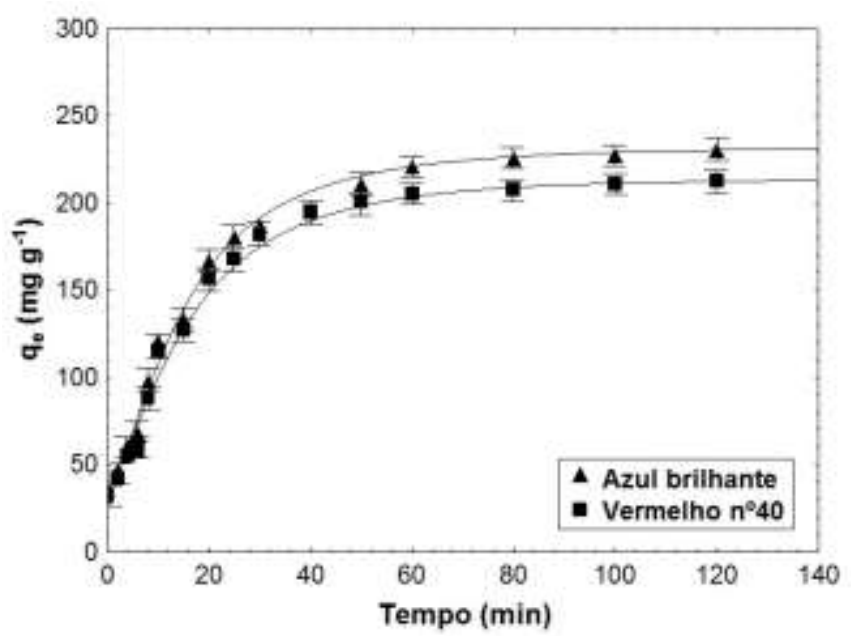

A partir da Figura 2 pode ser verificado que, para os dois corantes, o hidrogel scaffold apresentou um aumento brusco, na fase inicial, da capacidade de adsorção em relação ao 


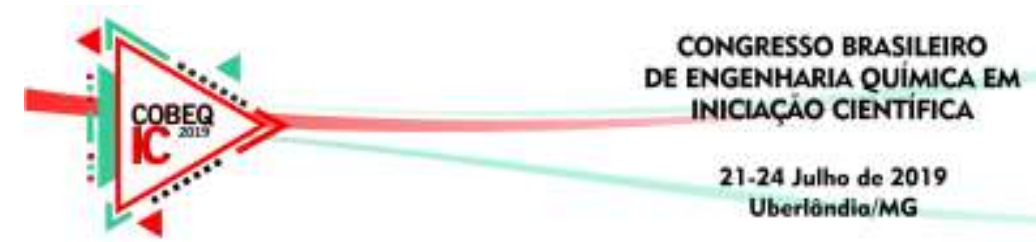

Uberlondia/MG

tempo, até a obtenção do seu equilíbrio. A capacidade de adsorção do corante azul brilhante se mostrou superior em relação ao vermelho ${ }^{\circ} 40$, podendo ser justificado devido ao número de grupos sulfonados presentes em sua estrutura. Além disso, pode ser observado que para ambos os corantes alimentícios verificou-se que o equilíbrio foi obtido em aproximadamente $50 \mathrm{~min}$.

Zhou et al. (2018) desenvolveram scaffolds contendo óxido de grafeno/quitosana onde foram testados para adsorção do corante cristal violeta, os autores apresentaram resultados relevantes para a remoção de corantes. Quando comparado ao presente estudo, o hidrogel scaffold com nanotubo se torna um adsorvente promissor de alta eficiência para adsorção de corantes em sistemas binários.

A Tabela 1 apresenta os parâmetros estimados dos modelos, R2 e o erro relativo médio (ERM), para os modelos cinéticos de Avrami e PPO.

Tabela 1- Parâmetros cinéticos para os modelos de Avrami e PPO.

\begin{tabular}{|lc|c|}
\hline Modelo & \multicolumn{2}{c|}{ Corantes alimentícios } \\
\hline Avrami & Vermelho $\boldsymbol{n}^{\boldsymbol{o}} \mathbf{4 0}$ & Azul brilhante \\
\hline$q_{a v}\left(\mathrm{mg} \mathrm{g}^{-1}\right)$ & 214,1 & 239,3 \\
\hline$k_{a v}\left(\mathrm{~min}^{-1}\right)$ & 0,055 & 0,099 \\
\hline$N$ & 0,801 & 0,823 \\
\hline$R^{2}$ & 0,99 & 0,99 \\
\hline$E M R(\%)$ & 3,9 & 4,3 \\
\hline $\boldsymbol{P P O}$ & & \\
\hline $\mathrm{q}_{1}\left(\mathrm{mg} \mathrm{g}^{-1}\right)$ & 222,3 & 248,7 \\
\hline$k_{1}\left(\mathrm{~min}^{-1}\right)$ & 0,068 & 0,110 \\
\hline$R^{2}$ & 0,98 & 0,99 \\
\hline$E M R(\%)$ & 6,4 & 5,8 \\
\hline
\end{tabular}

A partir da Tabela 1, observa-se que o modelo que melhor se ajustou aos dados experimentais foi o modelo de Avrami, devido aos altos valores do coeficiente de determinação $\left(R^{2}>0,95\right)$ e baixos valores do erro relativo médio $(E M R<6 \%)$.

\section{CONCLUSÃO}

Neste trabalho, foi avaliado desenvolvido e caracterizado o hidrogel scaffold de quitosana com nanotubo de carbono. Além disso, o comportamento cinético do hidrogel scaffold foi 


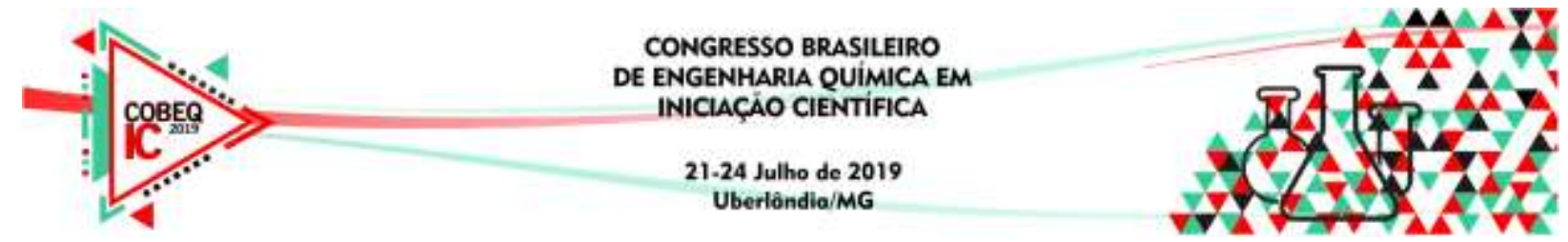

avaliado na adsorção dos corantes vermelho $\mathrm{n}^{\circ} 40$ e azul brilhante em sistema aquoso binário.

O hidrogel apresentou uma força do gel em torno de $735 \mathrm{~g}$, grau de intumescimento em torno de $91 \%$. As maiores capacidades de adsorção foram 239 e $214 \mathrm{mg} \mathrm{g}^{-1}$ para os corantes, azul brilhante e vermelho $\mathrm{n}^{\mathrm{o}} 40$, respectivamente. O modelo que melhor se ajustou aos dados experimentais foi o de Avrami.

\section{NOMENCLATURA}

\section{Símbolo}

$C_{0}$

$C_{t}$

$m$

$m_{i}$

$m_{f}$

V

\section{Definição}

Concentração inicial Concentração tempo $t$ na fase líquida

Massa de quitosana

Massa inicial do hidrogel

Massa final do hidrogel

Volume da solução
Unidade

$\left(\mathrm{mg} \mathrm{L}^{-1}\right)$

$\left(\mathrm{mg} \mathrm{L}^{-1}\right)$

(g)

(g)

(g)

(L)

\section{REFERÊNCIAS}

CRINI, G.; BADOT, P.M. Application of chitosan, a natural aminopolysaccharide, for dye removal from aqueous solutions by adsorption processes using batch studies: A review of recent literature. Prog. Polym. Sci., v. 33, p. 399-447, 2008.

CROISIER, F.; JÉRÔME, C. Chitosan-based biomaterials for tissue engineering. Eur. Polym. J., v. 4, p. 780-792, 2013.

GONÇALVES, J.O.; SANTOS, J.P.; RIOS, E.C.; CRISPIM, M.M.; DOTTO, G.L.; PINTO, L.A.A. Development of chitosan-based hybrid hydrogels for dyes removal from aqueous binary system. J. Mol. Liq., p. 225, v. 265-270, 2017.

JAYAKUMAR, R.; PRABAHARAN, M.; SUDHEESH, K. P. T; NAIR, S. V.; TAMURA, H. Biomaterials based on chitin and chitosan in wound dressing applications. Biotechnol Adv., v. 29, p. 322-337, 2011.

LUNA, M.S.; CASTALDO, R.; ALTOBELli, R.; GIOIELLA, L.; FILIPPONE, G.; GENTILE, G.; AMBROGI, V. Chitosan hydrogels embedding hyper-crosslinked polymer particles as reusable broad-spectrum adsorbents for dye removal. Carbohydr Polym., v. 177, p. 347-354, 2017.

TAN, K.B.; VAKILI, M.; HORRI, B.A.; POH, P. E.; ABDULLAH, A.Z., SALAMATINIA, B. Adsorption of dyes by nanomaterials: Recent developments and adsorption mechanisms. Sep. Sci. Technol., v. 150, p. 229-242, 2015.

VAKILI, M.; RAFATULLAH, M.; IBRAHIM, M.H.; ABDULLAH, A.Z.; SALAMATINIA, B.; GHOLAMI, Z. Chitosan hydrogel beads impregnated with hexadecylamine for improved reactive blue 4 adsorption. Carbohydr. Polym., v. 137, p. 139-146, 2016.

WESKA, R.; MOURA, J.M.; BATISTA, L.; RIZZI, J.; PINTO L.A.A. Optimization of deacetylation in the production of chitosan from shrimp wastes: Use of response surface methodology. J. Food Eng., v. 80, p. 749-753, 2007. 